\title{
Urbanisme, société et mobilité durable en
}

\section{Allemagne}

Society and sustainable mobility in Germany

Gesellschaft und nachhaltige Mobilität in Deutschland

\section{Anne Hecker}

\section{(2) OpenEdition}

Journals

Édition électronique

URL : http://journals.openedition.org/rge/939

DOI : $10.4000 /$ rge.939

ISSN : 2108-6478

Éditeur

Association des géographes de l'Est

Édition imprimée

Date de publication : 1 janvier 2007

ISSN : 0035-3213

\section{Référence électronique}

Anne Hecker, «Urbanisme, société et mobilité durable en Allemagne », Revue Géographique de l'Est [En ligne], vol. 47 / 1 | 2007, mis en ligne le 22 décembre 2009, consulté le 08 septembre 2020. URL : http://journals.openedition.org/rge/939 ; DOI : https://doi.org/10.4000/rge.939

Ce document a été généré automatiquement le 8 septembre 2020.

Tous droits réservés 


\title{
Urbanisme, société et mobilité durable en Allemagne
}

\author{
Society and sustainable mobility in Germany \\ Gesellschaft und nachhaltige Mobilität in Deutschland
}

\author{
Anne Hecker
}

\section{Introduction}

1 La mobilité urbaine constitue un vaste enjeu contemporain. L'une des principales vocations des villes réside en effet dans la connexion des activités humaines et dans l'intensification des échanges qu'elles favorisent. Urbanisation et mobilité sont donc intimement liées, les excès de l'une rejaillissant infailliblement sur l'autre. Ainsi, l'extension contemporaine de la ville produit des effets pervers sur les déplacements, et les excès automobiles qui en découlent asphyxient la ville en retour. Pourtant, l'Homme urbain est avant tout un être de mobilité, mobilité qu'il considère comme un droit et une nécessité et en aucun cas comme une contrainte à combattre. L'ennemi d'une urbanisation plus durable réside davantage dans « l'automobilité » (Chalas, 2001) et ses effets induits, que dans la possibilité offerte aux citadins de se déplacer librement. Dès lors l'Allemagne en général, et certains Länder tels que le Bade-Wurtemberg plus particulièrement, soutenus par une opinion déjà sensibilisée aux questions écologiques, travaillent à poser le problème de la ville contemporaine, et à réconcilier mobilité et urbanisme dans une optique de durabilité.

2 Ainsi, après s'être demandé si la mobilité urbaine peut contribuer à un urbanisme plus durable, notamment par le renouveau des modes de déplacement doux et ferroviaires, elle s'interroge désormais sur la contribution que l'urbanisme pourrait apporter à une mobilité urbaine durable. Cette évolution traduit également une autre avancée de la pensée urbanistique et des transports allemande: le glissement des politiques sectorielles simples vers des politiques intégrées, qui prennent non seulement en compte les différents modes de transport et leur complémentarité, mais également les 
multiples paramètres de la vie et de l'économie urbaine et leurs influences sur les déplacements des citadins.

3 Nous ferons dans cet article exception des nouveaux Länder et de Berlin, en raison des spécificités historiques de leur développement urbain. Les phénomènes de périurbanisation étaient en effet fortement contenus sous le régime est-allemand, et ne sont apparus qu'après la réunification du pays. On y a alors assisté à une véritable explosion urbaine (Wiessner 2001), à l'époque même où les villes ouest allemandes prenaient conscience des dérives de l'exurbanisation, et commençaient à œuvrer contre ses effets. Ce décalage de l'évolution urbaine entre les deux Allemagne justifie l'éviction de la partie orientale, mais ouvre la porte à une analyse de son évolution future.

\section{La mobilité urbaine peut-elle contribuer à un urbanisme durable?}

\section{A. Une morphologie périurbaine ouest allemande étalée et fragmentée}

Depuis l'époque moderne et les courants hygiénistes prescrivant d'aérer une ville médiévale repliée sur elle-même, jusqu'au génie urbain du XXe siècle venu affranchir les agglomérations des contraintes naturelles, les cités européennes ne cessent d'étaler leurs territoires, au détriment des zones rurales. Pression foncière, exode rural et accroissement naturel de la population ont décuplé la demande en espaces à urbaniser, et amorcé l'étalement de la tache urbaine. Par ailleurs, l'habitat contemporain traduit la prédilection croissante des citadins pour l'habitat individuel non mitoyen, à l'origine d'une dé-densification inexorable de l'espace urbain. Celle-ci s'opère au détriment des espaces périphériques, disponibles et moins onéreux, et dont l'accessibilité s'accroît avec la motorisation individuelle, l'augmentation des vitesses de déplacement et la multiplication des itinéraires routiers. Il en résulte la naissance de la Zwischenstadt ${ }^{1}$, un éloignement croissant des lieux de résidence du noyau urbain, ainsi que le développement de diverses zones d'activités à la marge des centres anciens, développés sur d'anciens terrains agricoles mis à disposition par la planification urbaine ellemême. Cette dernière n'a donc pas été en mesure de contrôler l'épanouissement de formes urbaines et de réseaux de déplacement susceptibles de concilier dynamique urbaine et développement durable.

Ce développement périurbain peu maîtrisé confronte aujourd'hui les villes européennes à un double phénomène d'étalement et de fragmentation spatiale et fonctionnelle. L'étalement se mesure à l'aune de l'espace utilisé par les activités humaines : en 2004, les surfaces bâties ou consacrées aux infrastructures de transport occupent $12,8 \%$ de la superficie allemande, en augmentation d'un point depuis $1996^{2}$. Depuis 1960, l'espace moyen dont chaque habitant des anciens Länder dispose pour se loger a ainsi triplé, passant de $14 \mathrm{~m}^{2}$ à $41,5 \mathrm{~m}^{2}$, entraînant à la baisse les densités de peuplement des espaces urbanisés. Parallèlement, chaque déplacement réalisé en véhicule particulier "consomme" un espace près de dix fois supérieur au même déplacement effectué à pied ou en transport en commun. Dès lors, les agglomérations ouest allemandes se caractérisent par de faibles densités de peuplement, alliées à des périphéries étalées, mais également fragmentées. En effet, les espaces péricentraux 
accueillent des équipements autrefois réservés aux centres-villes, qui répondent à une configuration monofonctionnelle jusqu'alors ignorée: après l'exurbanisation des activités industrielles vers des zones spécialisées, on observe l'apparition d'espaces périphériques distincts, dévolus à l'habitat, au commerce, aux loisirs ou aux services. Libérés des contraintes foncières des centres urbains, ils se caractérisent par un fort accroissement des surfaces consommées, tant par les bâtiments que par les équipements routiers ou dédiés au stationnement qui y sont associés.

6 Dès lors, l'extension et l'éloignement croissant de ces espaces périurbains hyper spécialisés et mono fonctionnels aboutissent à la généralisation d'une ville consommatrice d'espace et génératrice de déplacements de plus en plus nombreux, chaînés et lointains. Or l'orientation des politiques urbaines en vigueur lors de leur développement, plus favorables au mode routier qu'aux transports en commun, impose aujourd'hui à leurs utilisateurs le recours à l'automobile. Dès lors, cette dislocation accélérée de la structure urbaine place les agglomérations allemandes face à un accroissement inquiétant des multiples nuisances liées à l'automobile, au premier rang desquelles émerge la congestion urbaine - trop d'accessibilité tue l'accessibilité -, ellemême directement responsable de nuisances sonores, de pollutions et d'une consommation énergétique accrue.

7 Néanmoins, l'Allemagne se caractérise également par une conscience écologique traditionnellement implantée au cœur de la société: la préservation de l'environnement au profit des générations à venir est ainsi un principe essentiel intégré à la Constitution ${ }^{3}$. Dès la fin des années 1980, cette sensibilité a induit une prise de conscience de l'impasse dans laquelle s'engageaient les agglomérations allemandes, et a favorisé l'acceptation de mesures, parfois contraignantes, prises en faveur d'un développement plus durable. Un encadrement de l'extension urbaine par une planification plus directive que par le passé a favorisé la définition de limites de villes plus franches, et une évolution du regard porté par les citoyens allemands sur leurs moyens de transport. Les politiques sectorielles portant sur les déplacements nonmotorisés et le mode ferroviaire évoluent ainsi aujourd'hui vers des réflexions sur la gestion globale des déplacements urbains et périurbains.

\section{B. Les déplacements non motorisés}

8 Les actions en faveur du renouveau des mobilités les plus douces sont les plus emblématiques mais également les plus anciennes4. Depuis les années 1970 et les premières démarches en leur faveur, les aménagements cyclables ont plus que doublé jusqu'à atteindre $30000 \mathrm{~km}$ en 2001, dont $5000 \mathrm{~km}$ de bandes cyclables et $1500 \mathrm{~km}$ de pistes le long des routes nationales. L'obligation faite depuis 1981 d'adjoindre des bandes ou des pistes cyclables à toute nouvelle route a permis la réalisation de pistes bidirectionnelles le long d'un tiers du réseau routier fédéral. Diverses mesures sectorielles incitent à la pratique cyclable au quotidien, comme l'ouverture aux cyclistes des réductions d'impôts au titre des migrations pendulaires, jusqu'alors réservées aux seuls automobilistes, ou les différents amendements au Code de la Route tels que l'ouverture des couloirs de bus et des rues à sens unique à la circulation cycliste à contre-sens.

9 En 2002 est entré en vigueur un Plan National d'organisation de la circulation cycliste ${ }^{5}$ afin d'en accroître significativement la part modale dans les 10 années à venir, avec en 
point de mire les Pays-Bas et leurs $27 \%$ de part modale attribués au vélo. D'importants crédits fédéraux (à hauteur de 100 millions d'Euros pour la seule année 2002, soit le double de l'année précédente) vont financer de nouvelles réalisations, ainsi que la rénovation et la mise en conformité des itinéraires et des équipements existants. Ces mesures englobent différents aspects découlant de la pratique cycliste, et dont l'absence peut se révéler un frein puissant à la mobilité douce : signalisation spécifique dans la circulation routière, garage à vélos dans les habitations et les équipements publics, possibilité d'emprunter les transports en commun avec son vélo... La reconnaissance de la place du vélo dans la vie quotidienne et son intégration à la chaîne des transports illustre un glissement des actions sectorielles qu'incarnaient les politiques «vélos» des années 1970, vers des politiques de transport davantage intégrées.

De fortes disparités se cachent en Allemagne derrière le chiffre officiel de $10 \%$ de déplacements cyclistes: certaines villes, à l'image de Wuppertal ou de Wiesbaden offrent l'image de mauvais élèves en terme de pratique cyclable ${ }^{6}$, tandis que Brême, Fribourg-en-Brisgau ou Erlangen frôlent ou dépassent déjà les $20 \%$ de part modale, Münster atteignant même 32 \% (figure 1). Des politiques sectorielles fortes y ont fait leurs preuves, multipliant les aménagements routiers à destination des cyclistes. Mais leur réussite tient aussi à leur prise en compte de l'ensemble des besoins des cyclistes, au-delà de la réalisation de bandes cyclables isolées: de véritables parcours continus quadrillent ces cités, adjoints de l'interdiction du centre-ville aux véhicules motorisés, et de la mutation des secteurs résidentiels en Verkehrsberuhigte Bereiche (cours urbaines) : calqués sur le modèle néerlandais de la Woonerf, les piétons et les cyclistes y sont prioritaires sur les véhicules à moteur, tolérés au rythme de la marche. Ces villes présentent aujourd'hui de véritables "systèmes vélo", à l'image du "système voiture ", où chaque cycliste bénéficie d'un ensemble d'aménagements performants, du réseau continu de cheminements sécurisés au stationnement dédié et aux parkings surveillés, en passant par un système d'information spécifique, une signalisation adaptée ou l'installation de douches sur les lieux de travail. 
Figure 1 : Part modale des transports en Allemagne.

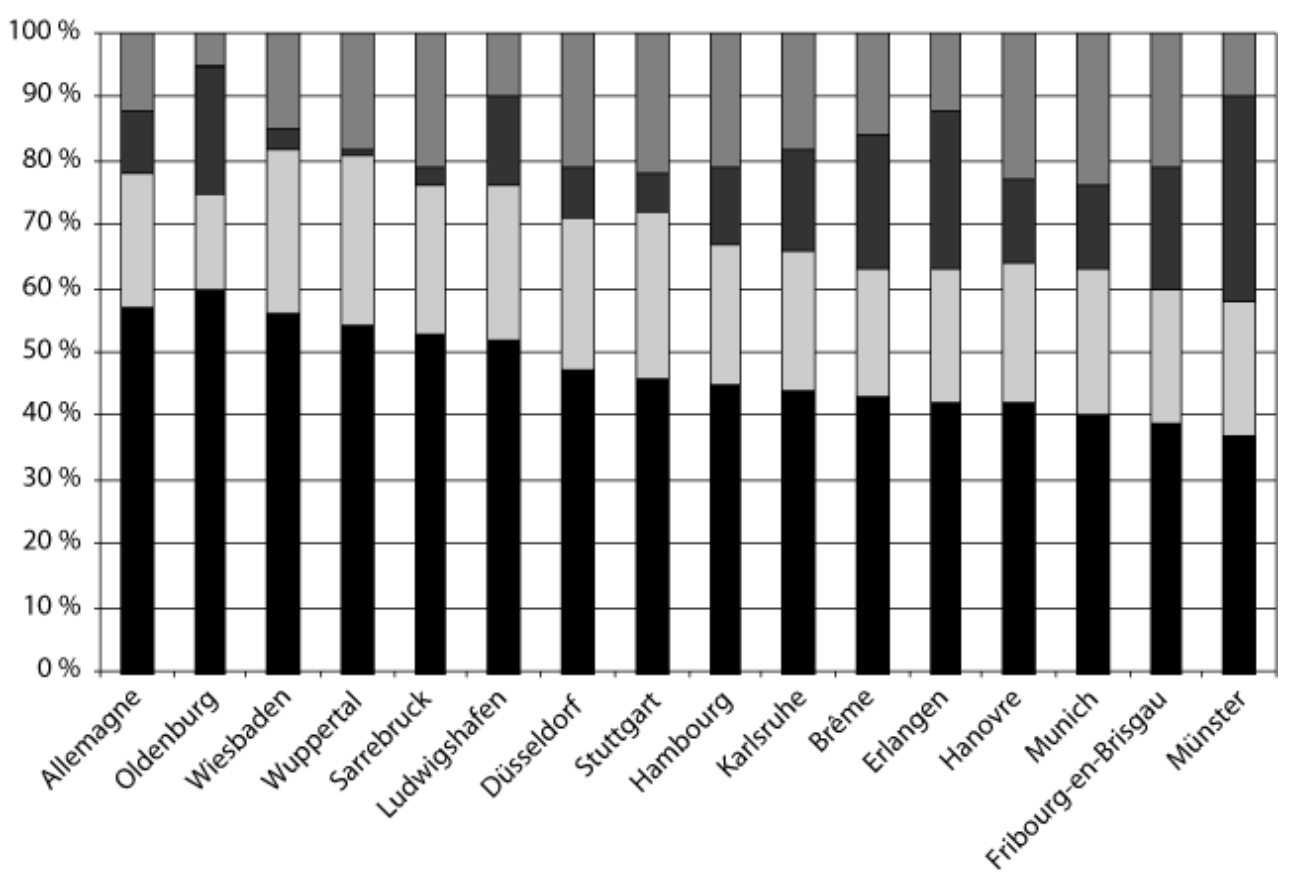

Socialdata 2002

- Voiture/moto Marche Vélo a Transports en commun

11 Toutefois, l'augmentation de la part des déplacements cyclistes n'induit pas forcément une amélioration des conditions de circulation dans la ville: si le report vers la bicyclette se fait aux dépends de la marche à pied ou des transports en commun, et non de la voiture individuelle, le bilan pour l'environnement urbain demeure inchangé. C'est ainsi le cas de Hambourg, où certes la part modale du vélo s'est accrue de 4 points entre 1976 et 1991, mais où les déplacements à pied ont dans le même temps chuté de 16 points, au bénéfice de la voiture, en augmentation de 13 points. Dès lors, les exemples qui seront développés par la suite (Stuttgart, Fribourg-en-Brisgau...) établissent que les agglomérations qui parviennent à réduire de manière significative le recours à la voiture individuelle se distinguent par une politique de transport globale, qui intègre dans une vision unique l'ensemble des déplacements des citadins et des périurbains, et gère activement la bonne articulation des différents moyens de transport. C'est notamment le cas de Fribourg-en-Brisgau qui, en dépit d'une augmentation de 25000 habitants et d'un doublement de son parc automobile (CERTU 2001), a enregistré la chute de 14 points des déplacements automobiles entre 1973 et 1993, liés à l'augmentation de 10 points des déplacements cyclistes et de 4 points des transports en commun. Les entrées de ville, où deux fois plus d'espace est dévolu aux déplacements alternatifs qu'à la circulation motorisée, traduisent bien cette prise en compte des différents modes de déplacement (photo 1), et notamment l'intégration du mode ferroviaire à la mobilité urbaine. 
Photo 1 : Le partage de la voirie à Fribourg-en-Brisgau.

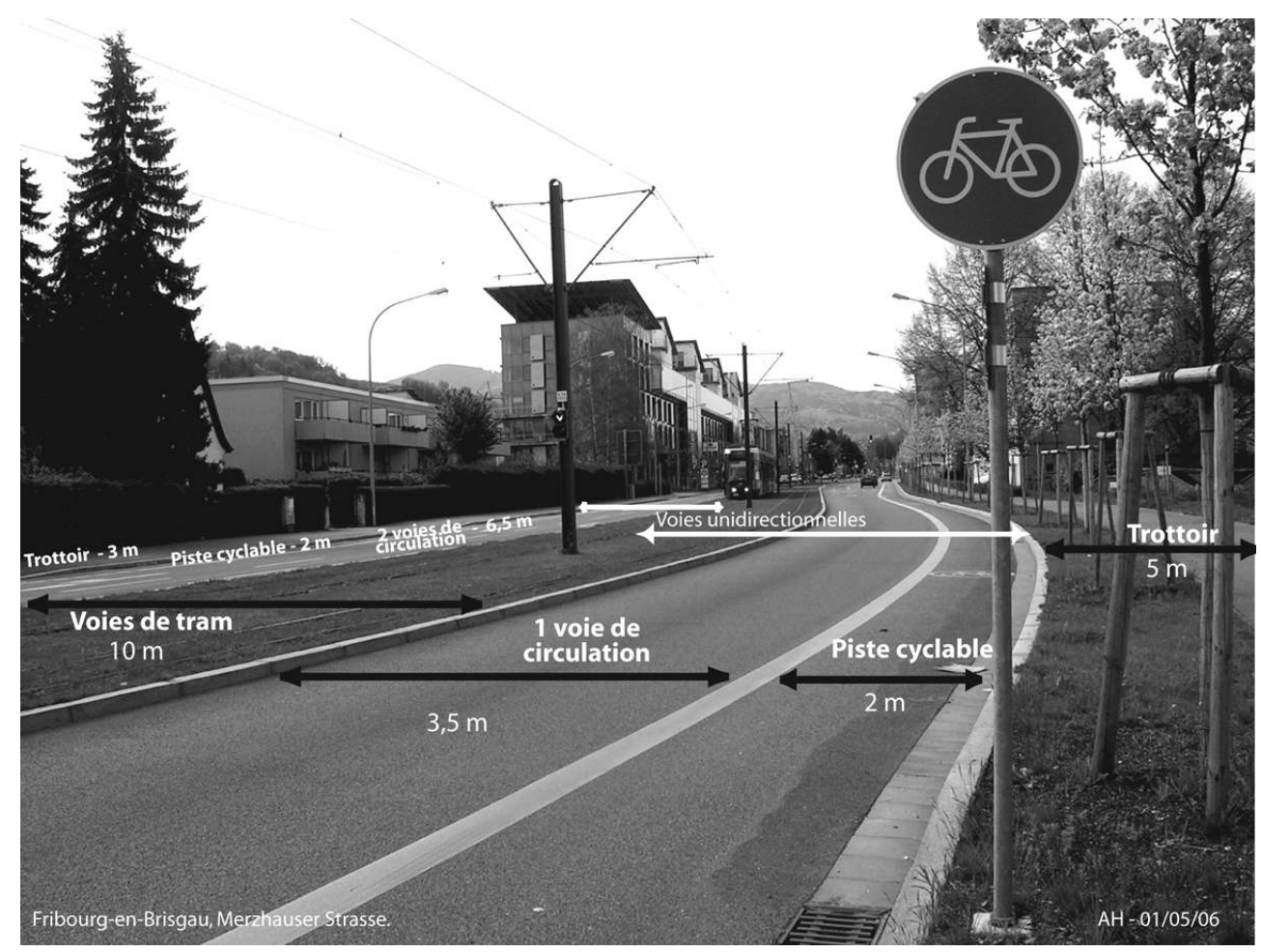

\section{Le renouveau des transports ferroviaires}

L'Allemagne a su préserver l'essentiel de ses réseaux de tramways urbains, à l'heure où les cités françaises s'efforçaient d'en faire disparaitre les traces de leurs paysages. Depuis les années 1980, elle a ainsi su diversifier l'offre et relancer la fréquentation de ses transports ferroviaires urbains et périurbains.

Karlsruhe fut ainsi le creuset de ce qui apparut en 1992 comme une solution « miracle » aux problèmes de transport urbain ${ }^{7}$. Le tram-train, qui s'apparente à un tramway desservant l'espace périurbain par l'emprunt du réseau ferroviaire classique, a alors connu un certain engouement. Son coût et les difficultés techniques qu'il présente (Van der Bilj et Kühn 2004) induisent désormais un glissement des projets vers la seconde génération de ce procédé. Le "train-tram ", où les véhicules ferroviaires circulent sur les plates-formes urbaines, procède d'un véritable retournement du concept, mieux adapté aux conditions de circulation locale comme l'illustre l'exemple de Zwickau. Les véhicules de son réseau urbain à écartement métrique ne pouvant circuler sur le réseau traditionnel, la pose d'un troisième rail sur la plate-forme urbaine a permis de proposer une liaison sans rupture de charge entre le centre-ville et les espaces périurbains grâce à des véhicules ferroviaires classiques.

Stuttgart illustre notamment le renouveau des transports ferroviaires dans les régions urbaines, et l'espoir d'un report modal de la circulation automobile vers les transports en commun. Ville-arrondissement de 600000 habitants, elle bénéficie, comme 12 autres grandes agglomérations, d'un Schnell-Bahn (S-Bahn), qui s'apparente au RER français : ses 6 lignes de chemin de fer traversent ville et banlieue, et accueillent des rames de voyageurs sur ses $174 \mathrm{~km}$ de rails en site propre. Physiquement séparées du reste du trafic ferroviaire, elles bénéficient de meilleures fréquences que le réseau traditionnel 
- une rame toutes les $15 \mathrm{mn}$ en heure de pointe -, d'arrêts fréquents - 69 stations soit un arrêt tous les $2,5 \mathrm{~km}$ en moyenne - mais très courts -1 minute au maximum. Son efficacité est relayée par les autres moyens de transport mis à disposition des voyageurs, notamment le Stadt-Bahn qui, depuis 1985, transforme progressivement les lignes de tramways existantes en desserte de métro léger à voie normale. Les lignes, prolongées vers les nouveaux quartiers de l'agglomération, sont réaménagées en site propre pour s'affranchir des encombrements routiers - en souterrain sous le centreville -, et proposer une bonne vitesse commerciale $(26,3 \mathrm{~km} / \mathrm{h}$ en moyenne) et des fréquences de passage élevées ( $6 \mathrm{mn}$ en heure de pointe).

Un funiculaire, un tram à crémaillère et un dense réseau d'autobus complètent l'offre de transports en commun, l'ensemble desservant de vastes parkings de dissuasion décentralisés : 15000 places de stationnement excentrées, couplées à des systèmes d'information orientent les automobilistes selon l'état du trafic et la saturation des parkings et participent à fluidifier la circulation intra muros. L'efficacité de ces aménagements de qualité se traduit par le report des Stuttgartois vers les transports en commun, auxquels ils ont recours pour un quart de leurs déplacements en général (+ 8 points depuis 1976), et pour $31 \%$ de leurs déplacements domicile travail, au détriment de l'automobile ( -5 points) et des modes non motorisés ( -3 points).

Sommés de résoudre la congestion urbaine chronique, les élus locaux et régionaux ont créé une institution métropolitaine, le Verband Region Stuttgart, qui oriente l'action en matière de transport du Verkehrs- und Tarifverbund Stuttgart. Ce dernier regroupe et coordonne les différentes entreprises de transport collectif de la Métropole. Chacune s'est ainsi vue attribuer une zone d'exploitation, définie selon ses capacités à la desservir efficacement et au moindre coût. Alliée à une intégration tarifaire de toutes les entreprises de transport de l'agglomération, la politique métropolitaine s'appuie donc sur leur complémentarité, excluant toute concurrence. C'est également le Verkehrs- und Tarifverbund Stuttgart qui établit - et impose - les grilles horaires de chacun, au service de la meilleure intermodalité possible, grâce à des correspondances rapides et facilitées par l'aménagement de plates-formes d'échange. L'exemple de la ville de Stuttgart illustre l'effort particulier porté à améliorer la qualité de ses dessertes, mais surtout à réaliser une bonne intégration de sa politique de transport.

Par ailleurs, la loi sur la régionalisation ferroviaire de 1996 incite les Länder à revitaliser les gares périphériques abandonnées ainsi que certaines dessertes de proximité, délaissées par la DB. L'objectif attendu est le financement par les Länder de nouveaux services de type RER, susceptibles d'ajuster l'offre à la demande ferroviaire et d'offrir de nouvelles opportunités de report modal aux périurbains. Quelques lignes ont ainsi déjà fait l'objet d'une modernisation, dont la réussite, à l'image du BOB (Bodensee Oberschwaben - Bahn, Bade-Wurtemberg) ou du Lipperländer (Westphalie, ILS 2001) participent du renouveau des transports ferroviaires de proximité. Ces derniers ont particulièrement été étudiés par le dispositif de recherches franco-allemand Bahn. $V_{i l l e}^{8}$, qui constate une nette augmentation de la fréquentation de ces lignes après leur régionalisation. En neuf années, la fréquentation du BOB a ainsi bondi de $370 \%$, les nouveaux clients émanant essentiellement des secteurs proches des arrêts desservis ( $52 \%$ demeurant à moins de $1 \mathrm{~km}$ et $80 \%$ à moins de $3 \mathrm{~km}$ et/ou $10 \mathrm{mn}$ de trajet). Parmi les conséquences constatées sur les zones desservies, Bahn. Ville note particulièrement la hausse des prix des terrains situés à proximité des arrêts desservis, et leur attractivité accrue pour l'implantation de nouveaux investisseurs. 

d'un nouveau rôle, celui de catalyseur d'un développement urbain « au bon endroit » et d'un mode de développement plus durable des agglomérations.

\section{L'urbanisme peut-il contribuer à une mobilité urbaine durable?}

19 En réaction à l'étalement urbain et à ses conséquences néfastes sur la mobilité, et conformément aux législations européennes, les agglomérations se tournent aujourd'hui vers de nouveaux modèles urbains. A l'aide des Agendas 21 locaux, et suivant notamment les recommandations du SDEC ${ }^{9}$, elles favorisent l'émergence d'une offre urbaine « aux bons endroits » d'une part (MASSKS / ILS 1999), et participe d'autre part à refaire sur elle-même une ville "des courtes distances", plus compacte mais également polycentrique.

\section{A. Ville compacte et polynucléaire : refaire sur elle-même une ville des courtes distances}

Pour certaines agglomérations allemandes, la recherche d'une "ville plus soutenable » passe par l'enrayement du mouvement centrifuge des habitants et des emplois, et par la reconquête de leurs espaces centraux. La définition d'une trame verte inconstructible cernant le centre limite un temps l'étalement urbain, mais sans autre mesure, l'exurbanisation au-delà de cette barrière s'avère inévitable.

Pour contraindre l'urbanisation à l'intérieur de ces limites, certaines agglomérations souhaitent, à l'image de Munich ou de Hanovre, densifier les espaces construits et élaborer une "ville compacte ». Rénovation, réhabilitation, requalification urbaine... ces agglomérations recomposent la ville sur elle-même, en recyclant espaces interstitiels ou vastes friches héritées de mutations ferroviaire, industrielle ou militaire, souvent bien situées. Par une offre nouvelle de logements urbains bon marché et ajustée à la demande contemporaine, ces communes s'efforcent de rendre à la ville son attractivité, et de limiter ainsi les velléités d'exurbanisation des jeunes ménages. A la réalisation de logements collectifs, elles associent le retour des grandes enseignes commerciales dans la ville, afin de juguler les échanges routiers avec la périphérie. L'Allemagne répond ainsi au concept de «villes des courtes distances », qui préconise l'implantation des services quotidiens à moins de $800 \mathrm{~m}$ des logements, de pôles d'échanges ou de parcs relais. L'intensification de l'usage de l'espace doit ainsi renforcer l'attractivité et la rentabilité des transports en commun, modérer à de courtes distances les besoins de mobilité et favoriser la pratique des déplacements non motorisés.

Toutefois, consciente des risques d'engorgement liés à une trop grande compacité des hommes et des activités, et face à la demande inflexible d'espace émanant de citadins favorables à une " urbanité en archipel $»^{10}$, l'Allemagne tend vers un modèle urbain certes plus dense, mais surtout polynucléaire et multifonctionnel. « Polynucléaire », car l'on assiste à la multiplication de nouveaux " centres ", créés ou adossés à des quartiers voire à des communes périphériques. La pression foncière s'en trouve répartie - et contenue - entre des quartiers ou des communes " périurbaines ", répondant ainsi aux 
besoins de logements liés à l'accroissement continu de la population urbaine, ainsi qu'à la demande en habitat moins dense. Toutefois, cette concentration urbaine localisée, qui tente de s'opposer à un étalement diffus de la tache urbaine, est strictement conditionnée par la présence ou à la réalisation de liaisons par des transports en commun efficaces, de type ferroviaire ou TCSP, et intègre impérativement une forte mixité fonctionnelle. Cette "multifonctionnalité » favorise la décentralisation et la redistribution des services, des activités, des équipements et des commerces, réinsérés au cœur d'un habitat certes périphérique, mais ainsi moins excentré. Elle s'accompagne de mesures contraignantes, bloquant l'installation en périphérie des activités de commerce et de loisirs, dévoreuses d'espace. Cette organisation doit alors minimiser les distances parcourues puisque tout ou presque se trouve dans son quartier, et favoriser les déplacements quotidiens non-motorisés ou le recours aux transports en commun.

La réussite de cette ville polynucléaire et multifonctionnelle se heurte toutefois à la liberté fondamentale du citadin de vivre sa "ville au choix » (Chalas 2001), et à rompre avec les préconisations de consommation émises par les urbanistes. Ces derniers ne peuvent contrer l'attractivité d'un commerce meilleur marché ou la réputation d'un cabinet médical, même implanté à l'autre bout de l'agglomération. Dès lors, pour limiter le recours à "l'automobilité » sans restreindre les désirs de mobilité de leurs citoyens, les agglomérations tentent d'organiser une "offre urbaine aux bons endroits ».

\section{B. L'offre urbaine « aux bons endroits »}

Les Länder et les agglomérations tentent de contrer la propension de la population à s'agglomérer autour des axes de transport privilégiés que sont les infrastructures routières, et de les inciter à se reporter vers les axes à privilégier que sont les transports en commun en site propre (TCSP) et les voies ferrées. Ainsi, lorsque s'est précisé le boom urbain, particulièrement sensible à l'Est de l'Allemagne, qui a suivi la réunification allemande, l'Etat a engagé un mouvement de réflexion autour des nouvelles extensions spatiales des villes. Cette réflexion s'est engagée tant dans les villes orientales qu'occidentales, selon un principe fondamental de la démocratie allemande : la concertation précède l'action. Cette bonne gouvernance favorise souvent l'acceptation de mesures contraignantes, mais dont la nécessité a été exposée et consentie. Un programme de 200 tables rondes locales a ainsi réuni pendant sept ans le Ministère, les villes, les districts, les agences d'urbanisme et les autorités organisatrices de transport, afin d'élaborer, d'expliquer et de lever les obstacles aux nouveaux principes devant régir les futures extensions urbaines. Ces dernières, selon le principe adopté de « l'offre urbaine aux bons endroits » doivent être implantées prioritairement à proximité immédiate de dessertes ferroviaires ou de TCSP, plus que jamais investis d'un rôle actif au service d'une urbanisation plus durable, qui assure en retour leur rentabilité par l'accroissement de leur clientèle potentielle.

Le Land de Rhénanie du Nord - Westphalie s'est particulièrement investi dans ce projet. Depuis 1995, le Baulandinitiative (initiative pour le développement urbain), renforcé depuis 1998 de nouvelles règles en matière de subventions, subordonne toute nouvelle construction aux transports en commun. Le Land a ainsi déterminé des pôles à urbaniser en fonction de leur accessibilité, tout grand projet d'habitat devant être réalisé à moins de $500 \mathrm{~m}$ d'un arrêt de TCSP ou $1000 \mathrm{~m}$ d'une gare, existants ou à créer. 
L'aide au logement, qui concerne 200000 domiciles dans le Land, exclut l'habitat en zone périurbaine, et n'est octroyé qu'aux résidences situées à moins de $1500 \mathrm{~m}$ d'un arrêt de TCSP ou d'une gare, ou bénéficiant de bus de rabattement vers ceux-ci. Diverses agglomérations ont décliné ces initiatives régionales, à l'image de Münster, dont le «schéma des initiatives territoriales" planifie l'évolution de la cité vers des pôles urbanisés équipés et " bien situés ", gelant toute urbanisation dans les secteurs ne répondant pas aux critères du Baulandinitiative. L'acquisition prospective et massive de terrains a fait de la Ville la propriétaire de la moitié des surfaces actuellement constructibles, qu'elle revend viabilisés et correctement desservis par les transports en commun, à des tarifs accessibles aux jeunes ménages. Entre 1990 et 2000, $75 \%$ des 17000 logements inaugurés étaient ainsi situés à moins de $1500 \mathrm{~m}$ d'une gare ferroviaire.

L'impact de la présence d'un arrêt de TCSP ou d'une gare ferroviaire sur les choix modaux des habitants de ces quartiers bien desservis a fait l'objet d'une évaluation par le Land (ILS 2001 b). On y constate qu'en l'absence d'une bonne desserte, seuls $7 \%$ des habitants d'un quartier recourent aux transports en commun. La présence d'un arrêt à moins de $1000 \mathrm{~m}$ de leur domicile convainc en revanche $24 \%$ des riverains à délaisser la voiture pour le train ou les transports en commun pour se rendre au travail. Si ce choix modal s'avère moins prégnant pour les autres déplacements (courses ou loisirs notamment), la facture énergétique liée aux transports s'avère toutefois réduite de $6 \%$ dans les quartiers bien desservis, illustrant l'impact positif du développement urbain directement conditionné à des transports collectifs efficients sur le développement durable des cités.

Au-delà de la Rhénanie, d'autres agglomérations ont adopté ce principe d'urbanisation, telle Langenhagen, ville banlieue de Hanovre (Basse-Saxe). Desservie par une liaison SBahn, elle a développé une politique incitative afin d'installer 7000 nouveaux habitants autour de l'arrêt communal d'ici 2010, illustrant l'une des conséquences locales de ce programme fédéral : la renaissance des quartiers de la gare. Le programme fédéral insiste également sur l'ouverture l'une à l'autre de la gare et de la ville, via la rénovation et la réintégration des espaces ferroviaires dans la cité. L'accessibilité est traitée en priorité, comme à Friedrichshafen, où l'arrière de la gare a été requalifié et intégré dans les cheminements piétonniers et cyclables. Ce quartier de friches ferroviaires, valorisées par la réalisation de commerces, de services de proximité, de logements et de bureaux, s'est ouvert à la gare et à la ville qui lui tournaient le dos : un souterrain a été prolongé sous la gare jusqu'aux nouvelles constructions, raccourcissant sensiblement l'accès aux quais et créant un lien avec le secteur commerçant du "bon côté » de la ville. Ainsi, la requalification urbaine des secteurs entourant les bâtiments ferroviaires, souvent délabrés et grevés de friches ou autres espaces en déshérence, offre l'image d'une ville qui se régénère en se reconstruisant sur elle-même.

La ville durable allemande régénère et adapte son tissu ancien aux pratiques actuelles de ses habitants afin de ramener ces derniers "au centre", centre de plus en plus multiple tant sur le plan spatial que fonctionnel. Cette mutation de la ville traduit l'évolution ultime des politiques urbaines, l'intégration: pour atteindre leur objectif d'une mobilité plus durable, elles prennent désormais en considération l'ensemble des paramètres qui influent sur elle, et interviennent de front dans tous les domaines 
concomitants - étalement urbain, localisation des services et des commerces, revitalisation des transports et de leurs « espaces de vie » ...

\section{Vauban : la réussite au prix d'une politique intégrée}

Le quartier Vauban, à Fribourg-en-Brisgau, illustre et synthétise les efforts menés par quelques agglomérations allemandes et par le land du Bade-Wurtemberg pour établir un urbanisme et une mobilité plus durables.

\section{A. Genèse d'un projet}

Cette ville du Bade-Wurtemberg constitue un écrin favorable à une expérience telle que Vauban: cité de 200000 habitants, au cœur d'une agglomération qui en compte le triple, elle se révèle tant la proie de la périurbanisation et de ses nuisances qu'investie dans les projets de développement durable. «Capitale écologique » de l'Allemagne, elle recense 10000 emplois directement rattachés aux activités environnementales, et abrite l'une des principales usines européennes de production de panneaux photovoltaïques. L'entrée des Grünen au Conseil Municipal dans les années 1970 n'est pas étranger à l'orientation de la politique fribourgeoise en matière de transport urbain, très favorable aux modes de déplacement doux et aux transports en commun. En dépit d'excellents résultats en matière de choix modal, la part de la voiture n'y excédant pas $39 \%$ (figure 1), les efforts de la municipalité portent aujourd'hui encore sur la déprise de «l'automobilité ». Mais au-delà, elle s'efforce désormais d'enrayer la fuite des jeunes couples vers de nouvelles périphéries, gagnées " auf der grünen Wiese ». Le projet de nouveaux quartiers à haute qualité environnementale, né dans les années 1990, s'est ainsi concrétisé dans deux secteurs: à l'ouest de la cité, le site d'une ancienne zone d'épandage des boues de station d'épuration accueille le premier, Rieselfeld, rapidement prolongé par le projet Vauban.

31 A $3 \mathrm{~km}$ du centre-ville, le long d'une voie ferrée aux services régionalisés, s'étendent en effet 38 ha des casernes, abandonnées par l'armée française en 1992. Cette friche militaire bordée de rails, véritable dent creuse au cœur d'une ville ceinturée d'espaces verts inconstructibles qui condamnent la ville à se développer sur elle-même ou en lointaine périphérie, apparaît comme une opportunité de densification à saisir. La municipalité se porte alors acquéreur de l'ensemble des terrains qu'elle dépollue et viabilise, et lance en 1995 le projet d'un nouveau quartier de 5000 habitants, fondé sur la mixité sociale et fonctionnelle, sur la haute qualité environnementale et sur la mobilité durable. La Ville engage immédiatement un processus de participation citoyenne, afin de définir un projet commun et de faire passer, par le consensus, ses exigences minimales, notamment en matière de transport. Ainsi naît en 1995 le Forum Vauban, entité de gestion et de coordination constituée d'associations citoyennes, d'experts pluridisciplinaires et de futurs propriétaires, qui intervient dès la planification dans 4 directions principales : promouvoir l'architecture écologique, les économies d'énergie et l'utilisation des ressources renouvelables; réduire l'imperméabilisation des sols et respecter la biodiversité ; favoriser la mixité sociale et fonctionnelle; et limiter le nombre d'automobiles dans le futur quartier en réduisant la place qui leur est dédiée ${ }^{11}$. 


\section{B. Objectif Zéro Voiture}

32 La médiation via le Forum Vauban a notamment permis à la municipalité d'imposer en douceur l'objectif emblématique d'un espace "Zéro Voiture », qui contracte de façon drastique la place accordée à la voiture. L'allée centrale et quelques rues périphériques sont certes accessibles à $30 \mathrm{~km} / \mathrm{h}$ et bordées de quelques places de stationnement payant. Mais les rues qui s'articulent de part et d'autre de ces arêtes sont en revanche des « cours urbaines ", où la voiture cède le pas aux piétons (photo 2 et figure 2). Aucun espace n'étant prévu pour stationner, seul l'arrêt temporaire permet de déposer courses et objets encombrants. Ce choix autorise un gain de place pour les logements et les espaces publics, mais impose aux habitants de Vauban d'opter pour l'une des trois solutions proposées : acquérir un logement en périphérie du quartier $(25 \%)$, seuls à être dotés d'une place de stationnement privative; accepter, comme $50 \%$ des habitants, d'acheter l'unique place de garage attachée à leur logement, au prix volontairement dissuasif de $17500 €$. Attachement légal mais pas physique, puisque cette place se situe dans l'un des 3 garages collectifs, implantés en périphérie du quartier, et accessibles par des cheminements piétonniers ou cyclables. Troisième possibilité, renoncer par écrit et pour une durée de 10 ans minimum à la possession d'une voiture individuelle, ce qui exempte le futur propriétaire de l'achat de la place de stationnement ( $25 \%$ des habitants). La limitation stricte à une seule voiture par famille et les difficultés de stationnement favorisent le recours au car-sharing : $46 \%$ des foyers ont ainsi adhéré à l'association Car Frei, qui gère un système d'autopartage entre résidents. Ses 63 voitures (une pour 20 adhérents), garées dans les garages collectifs, sont accessibles à tous moments aux adhérents, qui bénéficient en outre de tarifs préférentiels dans les transports en commun. 
Photo 2 : Cour urbaine du Quartier Vauban, Fribourg-en-Brisgau.

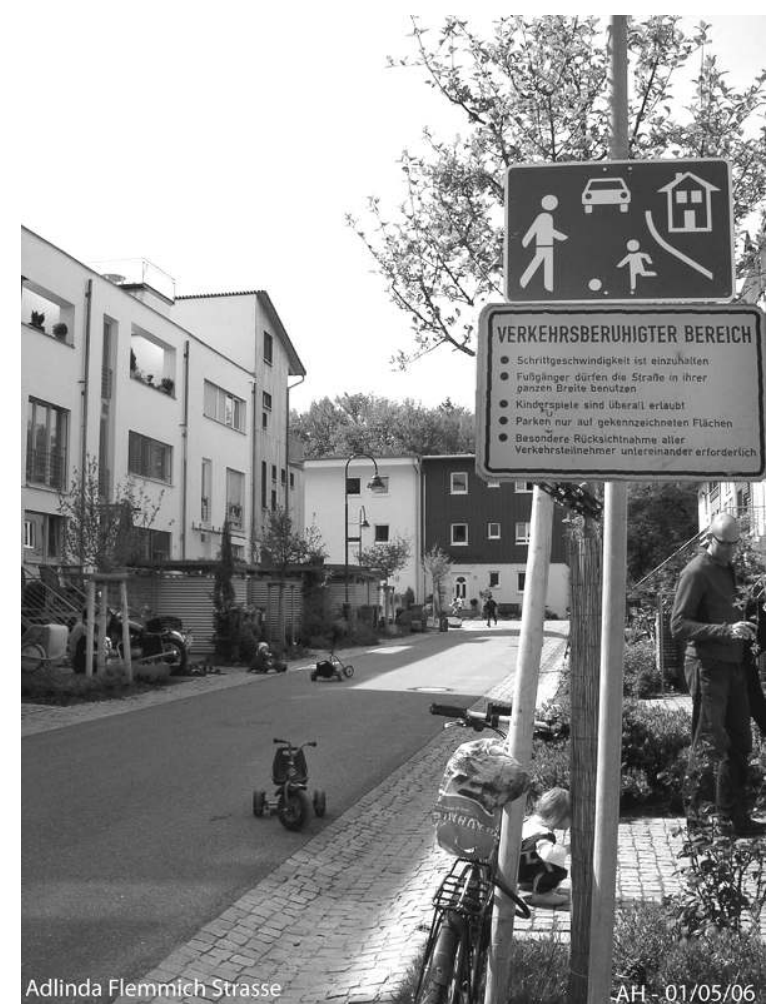

Entrée en « cour urbaine » (panneau à droite), qui offre la priorité aux piétons et aux jeux d'enfants, « autorisés partout ». Pas d'espace pour les voitures, mais des garages à vélo devant les immeubles, comme à gauche de l'image.

En échange de ces restrictions fermes, pour lesquelles on perçoit l'importance primordiale de négociations engagées dès la planification, la municipalité propose aux riverains des équipements susceptibles de rendre inutile le recours à la voiture: le quadrillage du quartier par des cheminements sécurisés, éclairés et stabilisés et donc accessibles à tous et par tous les temps, raccourcit les distances. A défaut de stationnement, chaque immeuble ou commerce dispose de garages à vélos fermés. Par ailleurs, le dispositif de transports en commun a été renforcé par la mise en service, le 29 avril 2006, de la ligne de Stadt-Bahn, amorcée dès 1995. Cette boucle qui relie le centre-ville à Vauban en $10 \mathrm{mn}$, selon une fréquence de 8 à 10 trams à l'heure en période de pointe, circule de $5 \mathrm{~h}$ du matin à minuit. Ce dispositif sera à terme complété par la création d'une halte supplémentaire sur la ligne de S-Bahn longeant le quartier, afin de favoriser le report vers le train des navetteurs parcourant de longues distances, notamment en direction de la Suisse. La mixité fonctionnelle, enfin, offre l'espoir d'un moindre recours à l'automobile : le quartier dispose d'une petite zone artisanale, qui concentre 600 emplois permanents, et des équipements de base: services publics (notamment liés à l'enfance, $75 \%$ des foyers comptant au moins un membre de moins de 18 ans), commerces de base (dont un supermarché) et espaces de loisirs se répartissent dans un périmètre de $700 \mathrm{~m}$ autour des habitations et sont donc accessibles à pied. 
Figure 2 : Schéma de l'organisation du Quartier Vauban à Fribourg-en-Brisgau

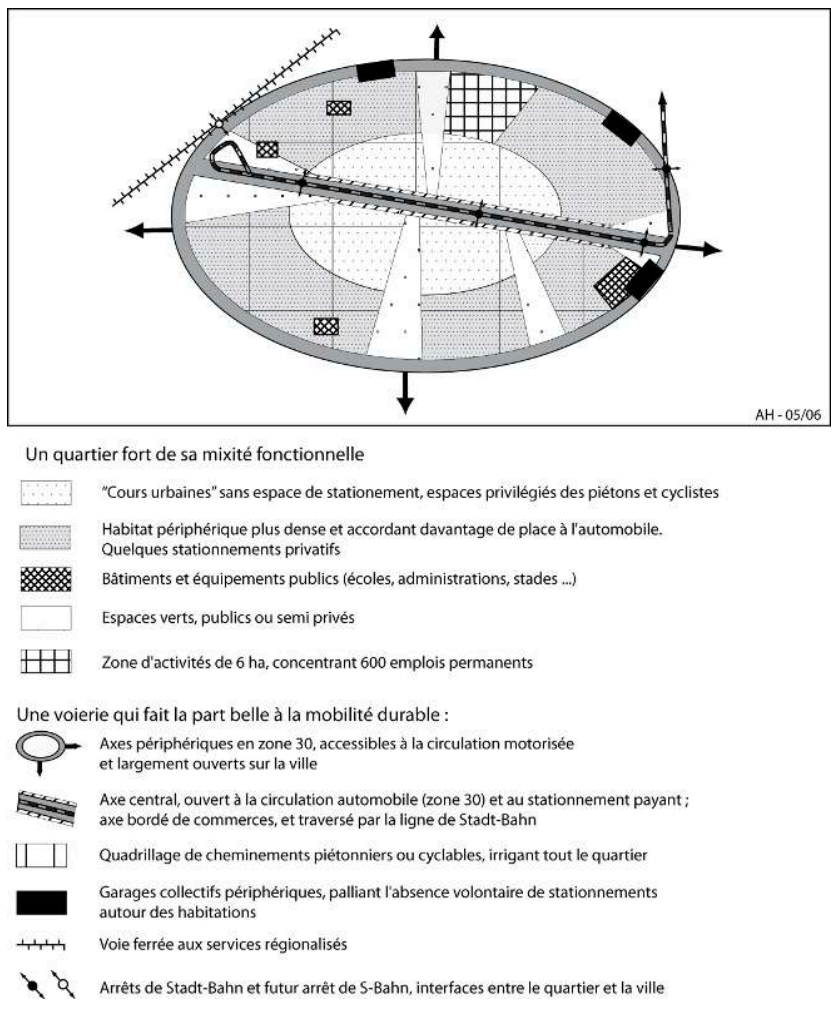

\section{Premiers éléments de bilan}

Le recul pour juger de la réussite d'un tel quartier demeure encore insuffisant, à l'heure où s'engage la dernière tranche de construction. Néanmoins, les premières données donnent à penser que les "pionniers » ont bien adhéré aux principes de durabilité du quartier. Ainsi, selon Scheurer (2001), les premiers résidents disposaient, à leur arrivée, de 247 voitures pour 1000 habitants (contre 588 en moyenne en Allemagne), 61 pour $1000 \mathrm{y}$ renonçant après leur installation. Le nombre de bicyclettes y connaît une évolution inverse, et l'on recense à Vauban 1108 bicyclettes pour 1000 habitants. Ces chiffres, qui se démarquent de la moyenne allemande, traduisent la sensibilité écologique des premiers emménagés et leur adhésion au mode de vie proposé. Quelques années plus tard, une étude de l'Institut d'Ecologie Appliquée (ARENE - IMBE 2005) réévalue la donnée à 450 véhicules pour 1000 habitants. La différence s'explique notamment par la réalisation précoce des logements sociaux autogérés et étudiants, dont les habitants, particulièrement convaincus par les principes du développement durable, prédominent dans l'échantillon analysé par J. Scheurer. En 2005 au contraire, les premiers logements périphériques dotés de places de stationnement sont achevés, et leur succès traduit l'attachement qui persiste, même à Vauban, pour «l'automobilité ». Néanmoins, aujourd'hui encore, moins de $30 \%$ des logements de Vauban disposent d'un emplacement de parking, alors que la statistique des quartiers périphériques allemands s'établit à $99 \%$...

La question se pose aujourd'hui des options qui seront retenues pour les constructions à venir. Globalement établies en périphérie nord du quartier, la possibilité s'offre aux promoteurs d'y inclure des emplacements privatifs de stationnement. Or, quelques 
signes de rébellion contre les normes strictes de Vauban sont apparus, notamment parmi les habitants de la troisième tranche de construction. Des incivilités liées à la mobilité y sont épisodiquement relevées, des résidents indélicats abandonnant leur voiture devant leur domicile, en dépit de l'interdiction et de l'inadaptation des ruelles. Les motifs avancés, notamment par les femmes seules et les parents d'enfants en basâge, font appel à l'éloignement des parkings collectifs, notamment lorsque le passage par le domicile est bref, que le résident est fatigué ou qu'il rentre de nuit. Un autre écueil réside dans le rejet de l'obligation qui est faite aux visiteurs de payer pour stationner leur véhicule, souvent loin, du domicile où ils sont invités.

Certains de ces problèmes devraient trouver leur solution avec la mise en service de la ligne de Stadt-Bahn, qui devrait capter une part des déplacements jusqu'alors automobiles. La fréquence des dessertes, la rapidité du trajet, l'inutilité de chercher une place de stationnement rare et chère, les correspondances étudiées et les tarifs attractifs offrent aux résidents de Vauban des arguments susceptibles d'orienter leur choix vers davantage de transports en commun. C'est ce qu'espèrent les acteurs de ce projet, qui comptent également sur la tradition de la négociation pour mettre un terme à ces incivilités et pour poursuivre l'expérience sans en dénaturer l'un des programmes phares.

Ces incivilités incarnent également l'un des aspects du principal risque qui guette cette expérience : la liberté des citadins du XXI ${ }^{\mathrm{e}}$ siècle. Cette politique urbaine exige de ses citoyens d'adhérer aux options prises pour eux par la municipalité et par ceux qui seront, dans quelques années, les " précédents résidents ». Certes, l'installation dans le quartier procède d'un choix, qui implique l'adhésion, en toute connaissance de cause, aux exigences qui constituent le fondement même de l'aménité des lieux. Mais les implications de ces options sont plus profondes que la simple possession d'un garage éloigné. Pour la municipalité, la réussite de l'expérience se mesurera à l'aune du renoncement définitif à la voiture, du report vers d'autres modes de déplacement, de la fréquentation prioritaire des commerces et services du quartier, et même de la convergence des emplois de la zone d'activité et des résidents. Mais ces employés souhaiteront-ils, ou pourront-ils, s'établir à Vauban? L'envie de "changer d'air » des habitants ne continuera-t-elle pas à les pousser à traverser l'agglomération pour consommer ailleurs? Et les destinations offertes par les transports en commun satisferont-elles ces résidents, ou souhaiteront-ils se rendre là où seule la voiture peut les mener? Au-delà d'un programme bien pensé et bien expliqué se pose la liberté fondamentale de l'être humain de choisir sa vie et sa ville. Pour que l'essai se transforme et marque le début d'une nouvelle ère urbaine, c'est donc une modification plus profonde de la société et de ses modes de vie qui s'avérera nécessaire.

\section{Conclusion}

Le Bade-Wurtemberg, et plus largement l'Allemagne, se trouvent-ils aujourd'hui, à travers diverses mesures et expériences telles que Vauban, sur le chemin d'une voie nouvelle pour la ville ? Et cette éventuelle nouvelle ère urbaine serait-elle transposable ailleurs qu'en Allemagne ou dans les pays du nord de l'Europe? Si ces Etats sont aujourd'hui à la pointe de l'expérimentation en matière d'urbanisme durable, c'est qu'ils réunissent des conditions particulières, qui font souvent cruellement défaut aux autres sociétés. La sensibilité écologique, la capacité à négocier, à mettre en place des outils de bonne gouvernance, et un degré de citoyenneté souvent au-dessus de la 
moyenne européenne autorisent notamment la réussite de telles expériences. Les structures politiques favorisent également la mise en place de telles expériences: la coopération intercommunale organisée au niveau de la région urbaine, offre notamment de grandes possibilités dans le champ de l'urbanisation et des transports, même si au final, peu d'agglomérations semblent en profiter (Adrian, 1996).

Comment imaginer leur reproduction, dans le contexte actuel de pays tels que la France ? L'émergence des SCOT, qui découlent de la loi SRU de décembre 2000, semble offrir un dispositif comparable d'intercommunalité à l'allemande, qui devra statutairement promouvoir l'équilibre entre l'urbanisation et la desserte des transports en commun. Ils présentent notamment l'avantage de dépasser les limites politicoadministratives habituelles, et notamment celles des agglomérations au-delà desquelles s'étend aujourd'hui la périurbanisation. Toutefois, les premiers SCOT mis en place tendent à suivre les périmètres existants des communautés d'agglomération ou des communautés urbaines, brisant de fait la dynamique qu'ils devraient favoriser. Néanmoins, ce dispositif demeure encore récent, l'essentiel des SCOT apparaissant encore en cours d'élaboration ou d'approbation. Les exemples étrangers réussis, notamment ceux du Bade-Wurtemberg, offrent des clefs, des pistes de réflexion à mettre au service d'expériences nouvelles à inventer et à adapter aux contextes locaux. Ils constituent également le témoignage des perspectives offertes par l'intégration des politiques urbaines et par les processus de participation citoyenne.

\section{BIBLIOGRAPHIE}

ARENE-IMBE (2005). - Quartiers durables, Guide d'expériences européennes, ARENE Ile de France et Institut Méditerranéen du Bâtiment et de l'Environnement, Paris, 146 p.

BAHN. VILLE (2003). - Rapport final, disponible en ligne : http://www.bahn-ville.net/fr/ 2_etapes/phase_9/livre.htm, 17 septembre 2006.

BELLANGER François, CROZET Yves, MASSOT Marie-Hélène, ORFEUIL Jean-Pierre, wIEL Marc (2005). - La Mobilité urbaine en débat, cinq scénarios pour le futur, Editions du CERTU, collection Débats $n^{\circ} 46,210$ p.

BORDREUIL Jean-Samuel (2000). - " La Ville desserrée », in La Ville et l'urbain, l'état des savoirs, Éditions la Découverte, Paris, 441 p.

CERTU (2001). - Les politiques cyclables en Europe, Tour d'horizon des politiques nationales, CERTU, collection Rapports, 77 p.

CHALAS Yves (2001). - Villes contemporaines, Editions du Cercle d'Art, Paris, 206 p.

HANNS Adrian (1996). - “Stadt und Region, Konzentration oder Dekonzentration”, in Stadtstrukturen - Status quo und Modelle für die Zukunft, Forum Zukunft Bauen, Beton- Verlag GmbH, Düsseldorf, pp. 50-56 
Institut für Landes- und Stadtentwicklungsforschung und Bauwesen des Landes NordrheinWestfalen (ILS) (2001). - Promouvoir l'urbanisation autour des dessertes ferrées et des TCSP : la politique du Land de la Rhénanie du Nord-Westphalie, Dortmund, ILS, 47 p.

Institut für Landes- und Stadtentwicklungsforschung und Bauwesen des Landes NordrheinWestfalen (ILS) (2001 b). - Mobilité et urbanisme, Habiter en périphérie près d'une desserte ferrée : quelle influence sur la mobilité et l'énergie ?, Dortmund, ILS, 63 p.

Ministerium für Arbeit, Soziales und Stadtentwicklung, Kultur und Sport des Landes NordrheinWestfalen (MASSKS) - ILS (1999). - Baulandentwicklung an der Schiene, Reihe Notiert $n^{\circ} 1325$, Düsseldorf

SCHEURER Jan (2001). - Urban ecology, innovations in housing policy and the future of cities : towards sustainability in neighbourhood communities, PhD Thesis, Murdoch University, $394 \mathrm{p}$.

SIEVERTS Thomas (2001). - Zwischenstadt, zwischen Ort und Welt, Raum und Zeit, Stadt und Land, Birckhaüser Verlag AG, Basel, 188 p.

Socialdata (2002). - Mobilität und Verkehrsmittelwahl in Deutschland, Erhebungen mit des KONTIV-Design, München, Socialdata, NN.

VAN DER BILJ Rob et KUHN Axel (2004). - “Tramtrain, the second generation”, new criteria for the "ideal tramtrain city", European Transport Conference (ETC 2004), Association for European Transport, Strasbourg, 4-6 octobre 2004.

WIESSNER Reinhard (2001). - « Développements périurbains en Allemagne de l'Est : l'exemple de la région de Leipzig », in Revue Géographique de l'Est, Tome XLI-2001 n³, Nancy, pp. 115-124.

\section{NOTES}

\section{SIEVERTS, 2001.}

2. Soit respectivement $45621 \mathrm{~km}^{2}$ en 2004 et $42052 \mathrm{~km}^{2}$ en 1996. Données du Statistisches Bundesamt Deutschland.

3. Article 20a de la Loi Fondamentale du 23 mai 1949, amendée par la loi du 26 juillet 2002.

4. Ces politique sectorielles concernent essentiellement la pratique de la bicyclette, la marche à pied n'étant pas directement favorisée mais plutôt associée à d'autres pratiques, notamment l'emploi des transports en commun.

5. “Fahr Rad! Nationaler Radverkehrsplan 2002-2012", Bundesministerium für Verkehr Bau und Wohnungswesen.

6. Respectivement 1 et $3 \%$ de part modale ... ce qui n'induit pas nécessairement un recours plus important à l'automobile. Toutes les parts modales citées dans l'article sont extraites de Socialdata, 2002.

7. Solution efficace, mais «miracle» tout relatif, la part modale des transports en commun de Karlsruhe ne s'étant accrue que de $2 \%$ entre 1992 et 2002, pour atteindre alors $18 \%$ de part modale.

8. Bahn. Ville, 2003 
9. Schéma de Développement de l'Espace Communautaire, Postdam, mai 1999.

10. Bordreuil 2000, évoquant la métaphore de Jean VIARD (La Société d'Archipel, éditions de l'Aube, collection Monde en cours, La Tour d'Aigues, 1998, 128 p.)

11. De multiples aspects innovants développé par le Quartier Vauban, notamment en terme de construction, d'énergie ou de mixité sociale ne seront pas ici abordés, car trop éloignés du sujet. Pour approfondir ces thèmes, on pourra notamment se référer à ARENE - IMBE 2005.

\section{RÉSUMÉS}

La ville du XXI ${ }^{\mathrm{e}}$ siècle souffre de son héritage périurbain. Face aux nuisances induites par la toute puissante "automobilité", les agglomérations allemandes présentent un regain de leurs dessertes ferroviaires urbaines et périurbaines. Tirant parti de cette réussite, elles s'efforcent d'orienter leur urbanisation vers des secteurs localisés prioritairement autour des dessertes ferroviaires ou de TCSP, au cœur d'un centre-ville en cours de redensification ou dans de nouveaux centres multifonctionnels plus éloignés. Le quartier Vauban, à Fribourg-en-Brisgau, offre ainsi l'exemple d'une expérimentation à haute qualité environnementale, fondée notamment sur de nouveaux concepts de mobilité urbaine intégrant un objectif « Zéro Voiture » et de fortes contraintes sociales.

Cities of the twenty-first century suffer from nuisance due to suburban development and invading "automobility". German urban-area shows a revival of urban and suburban railway services. They consequently try to establish new urban-area on places located around stations and along separated lanes of public transports. Those locations are elected among available places in downtowns to get it denser or in further multifunction centres in suburban areas. Vauban's district, in Freiburg-in-Breisgau, is an example of an environmental high quality experience, based on strong social constraints and new concepts about urban mobility, especially with one purpose : to build a car-free area.

Die Stadt des XXI. Jahrhunderts leidet unter dem Erbe der Suburbanisierung. Angesichts der Belastungen durch die Vormachtstellung der "Automobilität", erleben die deutschen Agglomerationen eine Wiederbelebung ihrer städtischen und suburbahnen Eisenbahnverbindungen. Von diesem Erfolg beflügelt, bemühen sie sich, ihre Siedlungsentwicklung auf Orte zu konzentrieren, die über eine gute Anbindung mit öffentlichen Verkehrsmitteln verfügen. Dies geschieht etwa im Zuge der Nachverdichtung der Stadtmitte oder auch in neuen, weiter außerhalb gelegenen multifunktionalen Zentren. Das Vauban-Viertel in Freiburg im Breisgau ist ein solches Beispiel für den Versuch, hohe Umweltqualität vor allem auf der Grundlage neuer Konzepte von Stadtmobilität und von sozialen Zwängen zu garantieren, hier in Form eines autofreien Stadtquartiers. 
INDEX

Mots-clés : Allemagne, mobilité urbaine, infrastructures de transport, urbanisation durable, mobilité durable, Fribourg-en-Brisgau

Keywords : Germany, urban mobility, transport infrastructures, sustainable urbanisation, sustainable mobility, Freiburg im Breisgau

Schlüsselwörter : Deutschland, städtische Mobilität, Verkehrsinfrastruktur, nachhaltige Stadtentwicklung, nachhaltige Mobilität, Freiburg im Breisgau

\section{AUTEUR}

ANNE HECKER

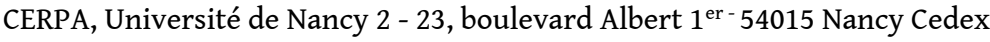

